\title{
Material Surface Design to Counter Electromagnetic Interrogation of Targets
}

\author{
H.T. Banks, K. Ito, G.M. Kepler, and J.A. Toivanen \\ Center for Research in Scientific Computation, \\ North Carolina State University, \\ Raleigh, North Carolina 27695-8205
}

December 8, 2004

\begin{abstract}
Utilization of controllable ferroelectric and ferromagnetic layers coating a conducting object to provide an attenuation capability against electromagnetic interrogation is discussed. The problem is formulated as a differential game and/or a robust optimization. The scattered field due to interrogation can be attenuated with the assumption of an uncertainty in the interrogation wave numbers. The controllable layer composed of ferromagnetic and ferroelectric materials $[9,10]$ is incorporated in a mathematical formulation based on the time-harmonic Maxwell equation. Fresnel's law for the reflectance index is extended to the electromagnetic propagation in anisotropic composite layers of ferromagnetic and electronic devices and used to demonstrate feasibility of control of reflections. Our methodology is also tested for a non-planar geometry of the conducting object (an NACA airfoil) in which we report our findings in the form of reduced radar cross sections (RCS).
\end{abstract}

\section{Introduction}

In this paper we discuss an optimal attenuation problem, i.e., we attempt to maximize attenuation capabilities of interrogating signals by utilizing a controllable dielectric layer on the surface of a conducting object. The objective of the interrogator is to detect and identify the location and shape of the conducting object based on the scattered field from an interrogation incident field, i.e., the solution of an inverse scattering problems $[2,6]$. In the

plane wave case the incident electromagnetic (EM) field has the form $\left(\vec{E}^{(i)}, \vec{H}^{(i)}\right) e^{i \vec{k} \cdot \vec{x}}$ and the 
interrogator has control over the wave numbers $\vec{k}$. The attenuation problem is to minimize or diminish the detection capability of interrogation by either grating [13] or fabrications on the object surface. In this paper we consider the utilization of thin controllable dielectric surface layers on the object as a method for achieving the attenuation capability. Here "controllable" means that we have the capability of adjusting the material properties of the surface layers parametrically and/or dynamically. The technical ideas developed here also have potential to aid in the design of medical shields employed to protect parts of an irradiated target or to focus radiation to pinpoint specific regions of the target.

In these initial investigations, we investigate an "active" design case in which one determines values of the dielectric permittivity and magnetic permeability of the controllable layer in order to attenuate reflections. From a control theoretic viewpoint this is a "passive" or open loop control strategy. But our efforts here lay the foundations for "active" or closed loop control strategies in which one combines controllable layer dynamics with a sensor for incoming interrogating signals to develop real time feedback controls for adaptive choice of the permittivity and permeability of the controllable layer.

To investigate feasibility, we first formulate the problem as a differential game. For example, we assume the time-harmonic incident EM plane wave is impinging on the surface at $z=0$ and we control the effective dielectric constant $\epsilon$ of the surface layer on top of the conducting material. The scattered field due to the interrogation can be evaluated based on the timeharmonic Maxwell equations [6]. In the case when the dielectric constant is homogeneous in the horizontal directions (planar geometry) the reflectance index $R=R(\vec{k}, \epsilon)$ is determined by Fresnel's law (see [14] and Section 4). Thus, the problem of nullifying the scattered field can be cast as the minimization of the scattering EM wave in terms of $|R|^{2}$, i.e.,

$$
\min _{\epsilon \in Q} \max _{\vec{k} \in K}|R|^{2}
$$

where $Q$ is a set of admissible dielectric constants and $K$ is a set of possible interrogation wave numbers. In order to determine the admissible set $Q$ we must describe controllable 
mechanisms of the dielectric layer. Thus, the problem of nullifying the scattered field can be formulated as min-max problem of minimizing the largest reflectance by interrogations over $\vec{k} \in K$, over all possible designs ( $\epsilon \in Q$ in this case). This game theoretic formulation is used in many other design problems. It does not assume any information on the uncertainty of interrogations and thus it may lead to a conservative design. An alternative formulation can be given in a more robust form, i.e.,

$$
\min _{\epsilon \in Q} \int_{K}|R|^{2} d \mathcal{K}(\vec{k})
$$

where $\mathcal{K}$ is a probability distribution function on the wave numbers $\vec{k}$. This formulation then needs information about the distribution function $\mathcal{K}$ of the interrogating plane wave. As demonstrated in Section 2, better knowledge of the distribution function greatly improves the performance of minimizing the scattered field.

After demonstrating attenuation capabilities, we turn to the general case of a non-planar conducting medium with a controllable coating layer. The far field pattern $F(\theta)$ of the scattered field (see, [6] and Section 5) is then a function of the wave number $\vec{k}$ of the incident plane wave and the material properties $(\epsilon, \mu)$ of the controllable layer, i.e.,

$$
F(\theta)=U(\vec{k},(\epsilon, \mu) ; \theta), \quad 0 \leq \theta \leq 2 \pi
$$

One can select the performance index $J(\vec{k},(\epsilon, \mu))=\Phi(F)$ to perform specific alternations of the scattered field, which of course depend on the inverse techniques employed by the interrogator. Here $\Phi(F)$ is some performance index for the far-field pattern $F(\theta)$. We investigate optimal radar cross sections (RCS) for one class of such problems.

We note that the existence of solutions to a general min-max problem is guaranteed under the condition that given $\epsilon \in Q$ the value function $V(\epsilon)=\sup _{\vec{k} \in K} J(\vec{k}, \epsilon)$ is lower semi-continuous, which is typically satisfied under very mild conditions (e.g, see [12]) when $Q$ is compact. The saddle point property of a solution pair $\left(\vec{k}_{0}, \epsilon_{0}\right)$ :

$$
J\left(\vec{k}, \epsilon_{0}\right) \leq J\left(\vec{k}_{0}, \epsilon_{0}\right) \leq J\left(\vec{k}_{0}, \epsilon\right) \text { for all } \epsilon \in Q, \vec{k} \in K
$$


holds locally if the Hessian of $J(\vec{k}, \epsilon)$ is hyperbolic at $\left(\vec{k}_{0}, \epsilon_{0}\right)$. The existence of solutions to the robust formulation then simply follows from the continuity of $J\left(\vec{k}, \epsilon_{0}\right)$ with respect to $(\vec{k}, \epsilon) \in K \times Q$.

A brief outline of our presentation here is as follows. In Section 2 we consider the exact problem for the planar geometry and present example numerical calculations for optimal design of the dielectric layer based on a robust formulation and demonstrate the feasibility of this approach and the effectiveness of the design. In Section 3 we discuss a controllable layer composed of ferromagnetic and ferroelectric materials as proposed by How and Vittoria $[9,10]$. This composite model is designed so that a control mechanism for the material properties of the layers can be achieved in both a parametric and a dynamic manner. In Section 4 we mathematically formulate the forward problem for the controllable composite layers, including the tensor permeability in the ferrite layer, by calculating the scattered field $R$ as a function of the interrogating wave and the near surface composition. We consider the time-harmonic case with plane wave interrogations of the planar geometry (i.e., the composite layers are homogeneous in $(x, y))$. In this case we construct the plane wave solution and an analytic expression for the reflectance index $R$. Another important feature of our formulation is the possible identification of the interrogating wave in terms of its distribution $\mathcal{K}$. Since the plane wave calculation also yields surface currents as an explicit function of the incident interrogations, the surface current measurements can, in principle, be used to identify the distribution $\mathcal{K}$ of the interrogations $\vec{k}$. In Sections 5-7 we present results for our formulation when applied to a non-planar geometry by considering the NACA0012 airfoil [15]. In general (and in particular in this case) we do not have an analytic expression for the far field pattern $F$ and thus we use a numerical computation of the scattered field. In this case, our numerical computations for the scattered field $F$ are implemented using the finite-element method (in Section 6). Our numerical findings are presented for an optimal homogeneous coating layer. 


\section{$2 \quad$ Feasibility study}

In this section we first demonstrate the feasibility of our approach. We consider an incident parallel-polarized $\left(\mathrm{TE}_{x}\right)$ plane wave $\vec{H}=\left(H_{x}^{(i)}, 0,0\right) e^{i \vec{k} \cdot \vec{x}}$ impinging on the interface of the first and second layers at $z=0$, as depicted in Figure 1. The interface between the second and third layers is located at $z=-d$, where the third layer is a perfect conductor. We control the effective dielectric constant $\epsilon^{(2)}$ of the second layer coating the conducting material. By Fresnel's law (see $[3,14]$ and the discussion in Section 4) we have that the reflection coefficient or reflectance index is given by

$$
R=\frac{\frac{\epsilon^{(2)} k_{z}^{(1)}-\epsilon^{(1)} k_{z}^{(2)}}{\epsilon_{z}^{(1)}+\epsilon^{(1)} k_{z}^{(2)}}+e^{-2 i k_{z}^{(2)} d}}{1+\frac{\epsilon^{(2)} k_{z}^{(1)}-\epsilon^{(1)} k_{z}^{(2)}}{\epsilon^{(2)} k_{z}^{(1)}+\epsilon^{(1)} k_{z}^{(2)}} e^{-2 i k_{z}^{(2)} d}},
$$

where

$$
\begin{aligned}
& k_{z}^{(1)}=\frac{2 \pi}{\lambda} \sqrt{1-\sin ^{2} \varphi_{0}} \\
& k_{z}^{(2)}=\frac{2 \pi}{\lambda} \sqrt{\epsilon^{(2)}-\epsilon^{(1)} \sin ^{2} \varphi_{0}} .
\end{aligned}
$$

Here $\varphi_{0}$ is the incident angle with respect to the normal to the surface $\left(\tan \varphi_{0}=\frac{k_{y}^{(1)}}{k_{z}^{(1)}}, k_{x}^{(1)}=\right.$ 0 ) and $\lambda$ is the wavelength of the incident wave. We note from (2.1) that $R$ depends on the ratio $\epsilon^{(2)} / \epsilon^{(1)}$ and hence without loss of generality we may normalize the parameters so that $\epsilon^{(1)}=1$. The reflectance index $R$ is a function of $\left(\lambda, \varphi_{0}\right)$, the normalized dielectric constant $\epsilon^{(2)}$, and the thickness $d$ of the surface layer. We assume that $d$ is positive and fixed. We parameterize the incident wave in terms of $\left(\lambda, \varphi_{0}\right)$.

In Figures 2 and 3 we depict the robustness of the optimal solution by plotting the reflectance intensity $|R|$ as a function of the incident angle and the normalized thickness/wave length ratio, defined as $a=\frac{d}{\lambda}$. In Figure 2 we assume that the uncertainty in wave numbers is only due to uncertainty in the incident angle $\varphi_{0}$, which is uniformly distributed on the interval $[36,54]$ degrees, and graph the intensity $|R|$ corresponding to the optimal dielectric constant 


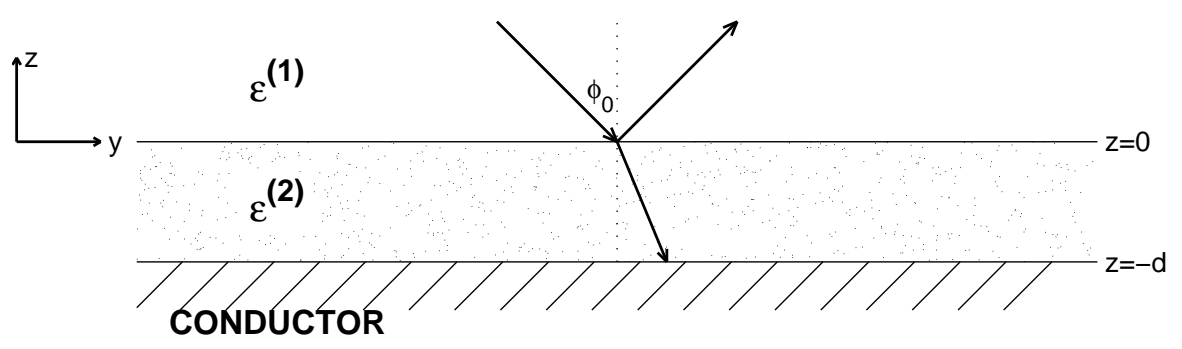

Figure 1: Schematic representation of the reflection of a plane wave incident with angle $\phi_{0}$ on a planar three layer stack. The top two layers are dielectric media. The third layer is a perfect conductor.

$\epsilon^{(2)}=1.008+.693 i$ as a function of the incident angle. The integration of $|R|^{2}$ over $\varphi_{0}$ is performed using Simpson's rule. The intensity of the reflection is well attenuated over the uncertainty interval $[36,54]$ degrees.

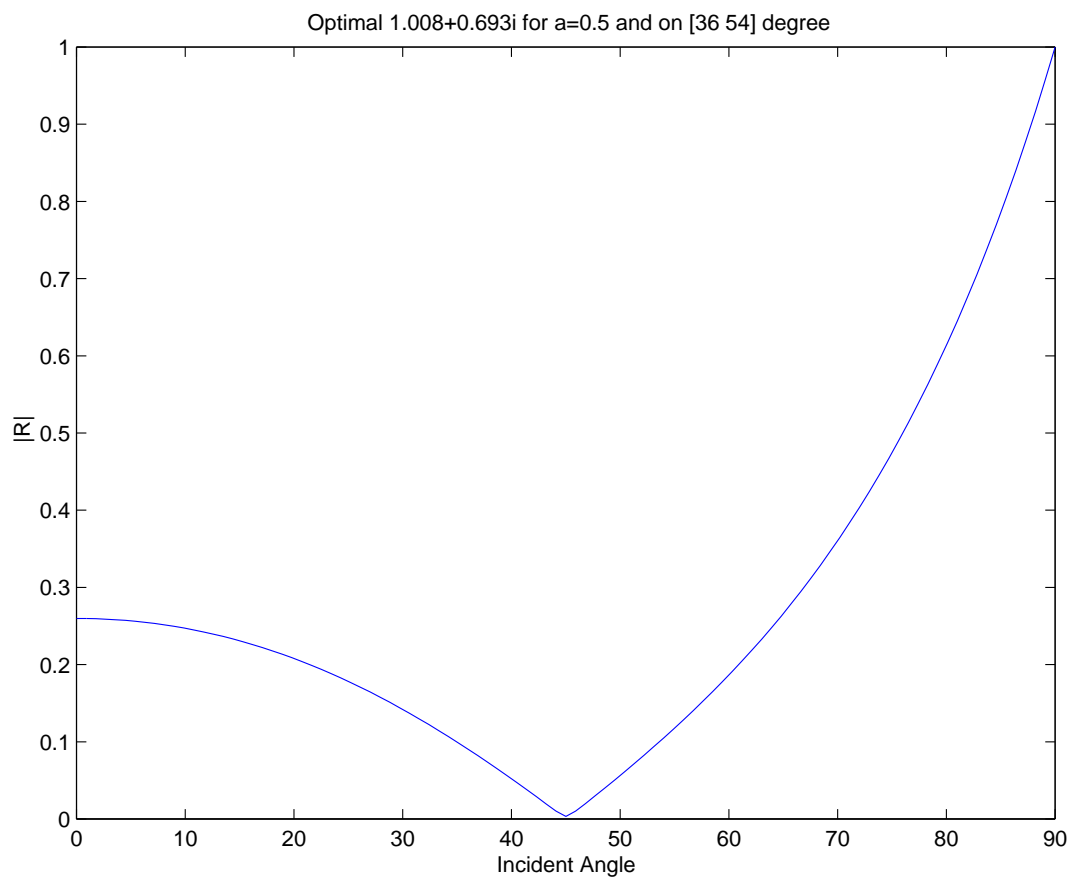

Figure 2: The reflected intensity $|R|$ as a function of the incident angle $\varphi_{0}$. The uncertainty interval is $[36,54]$ degrees.

Next, we assume that there is uncertainty in both the incident angle $\varphi_{0}$ and the normalized thickness/wave length ratio $a$, which are uniformly distributed on a rectangle [36, 54] $\times$ $[0.3,0.7]$. In Figure 3 we plot the intensity $|R|$ corresponding to the optimal dielectric constant $\epsilon^{(2)}=1.4309+1.0724 i$ for several sampled incident points in the frequency. A 
reasonable attenuation over the uncertainty box is obtained in this example. It is clear that the performance depends on the quality of the information on the distribution of the interrogating wave, as demonstrated by the better attenuation results in the first case (Fig. 2) than in the second case (Fig. 3).

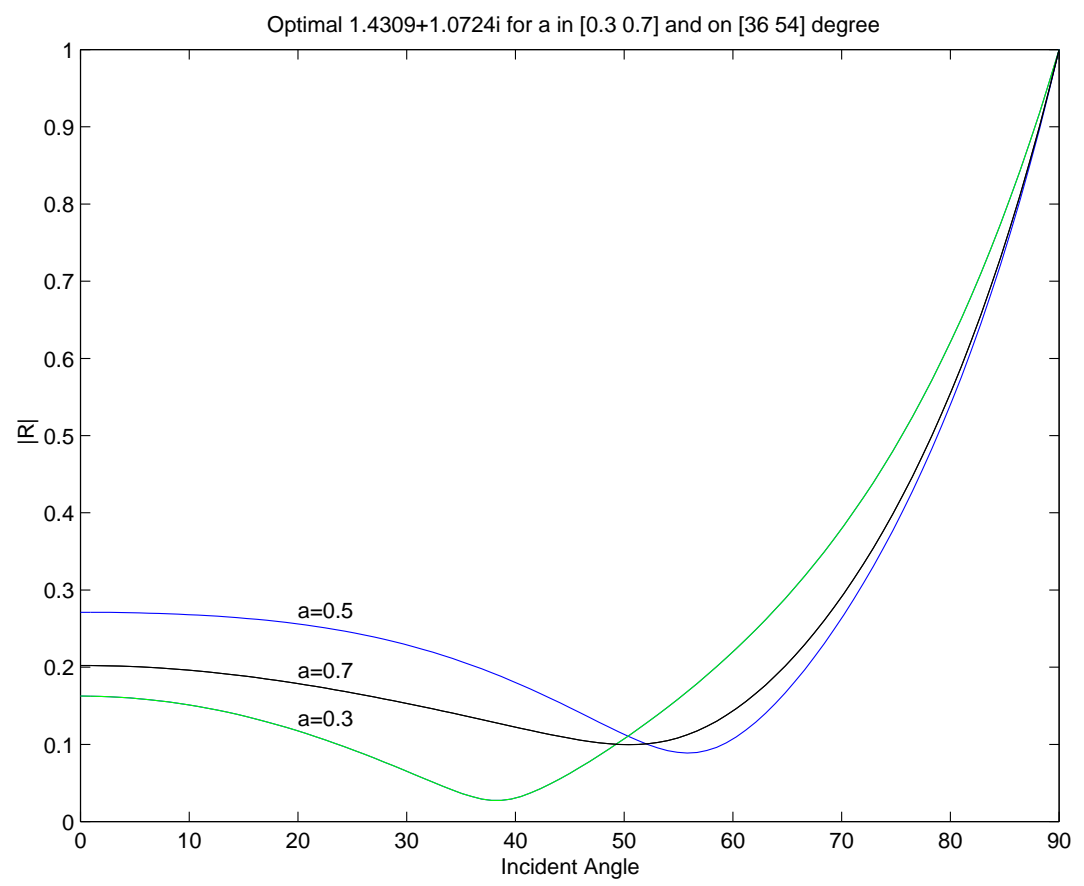

Figure 3: The reflected intensity $|R|$ as a function of the incident angle $\varphi_{0}$ and the normalized thickness/wave length ratio $a$. The uncertainty box is $[36,54] \times[0.3,0.7]$ 


\section{Controllable sublayers composed of ferromagnetic and ferroelectric materials}

In this section we describe an experimental device that can be used to control the dielectric permittivity (and magnetic permeability) in a coating layer as discussed in the previous sections. In Figure 4 we present a schematic of the configuration of an active reflecting device proposed and investigated experimentally by How and Vittoria in [9, 10]. The reflector contains a ferrite layer and a ferroelectric layer as constituents. The permanent magnet provides a common magnetic bias so that the ferromagnetic resonance (FMR) condition can be readily achieved and thereby facilitate sensitive magnetic tuning by the local Helmholtz coils. The dielectric properties of the ferroelectric layer are controlled through the ground plane bias field. The purpose of this reflector design is to provide phase and impedance control of the composite layers so that nullification and alteration of the scattered wave can be achieved in the response to an incident interrogating EM wave. The integrated circuits are designed so that the tuning sensitivity of the device is enhanced. The key element of the device is that the material properties $\mu(H)$ and $\epsilon(E)$ of the composite layers are controllable in terms of the magnetic mean in the ferrite layer and the electric mean in the ferroelectric layer, and thus can support agile frequency attenuation.

The most important device characteristic of the ferrite in our study $[9,10]$ is that the magnetic permeability $\bar{\mu}$ is a tensor, so that, due to the gyromagnetic effect, EM propagation in the ferrite is anisotropic in the presence of a dc-bias magnetic field $[11,19]$. For a ferrite magnetized in the $y$ direction with damping and no demagnetization, the permeability tensor is given by [16]

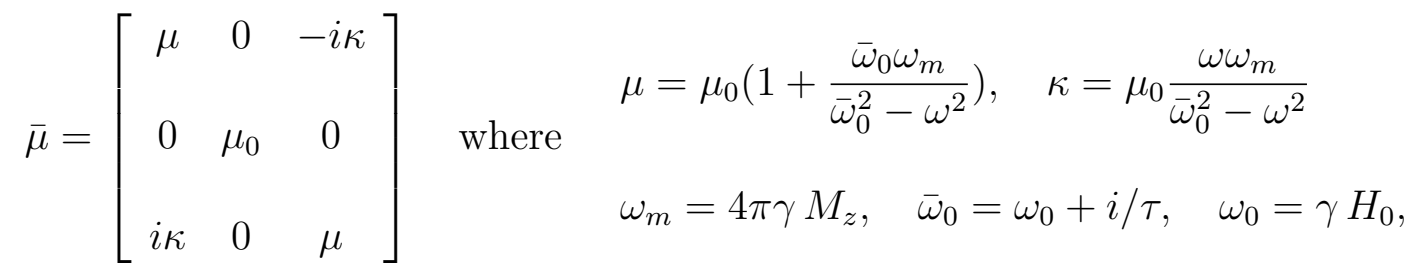

$\omega_{0}$ is the precession frequency, $H_{0}$ is the impressed d.c. magnetic field, $\gamma$ is the gyromagnetic 
ratio, $M_{z}$ is the saturation magnetization, and $\tau$ is the relaxation time.

The ferrite device is most useful if it operates near the FMR frequency $\omega_{0}$ so that the rapid change in magnetic permeability can be effectively utilized, either to obtain frequency-tuning capability or to remove the degeneracy between modes $[9,10]$.

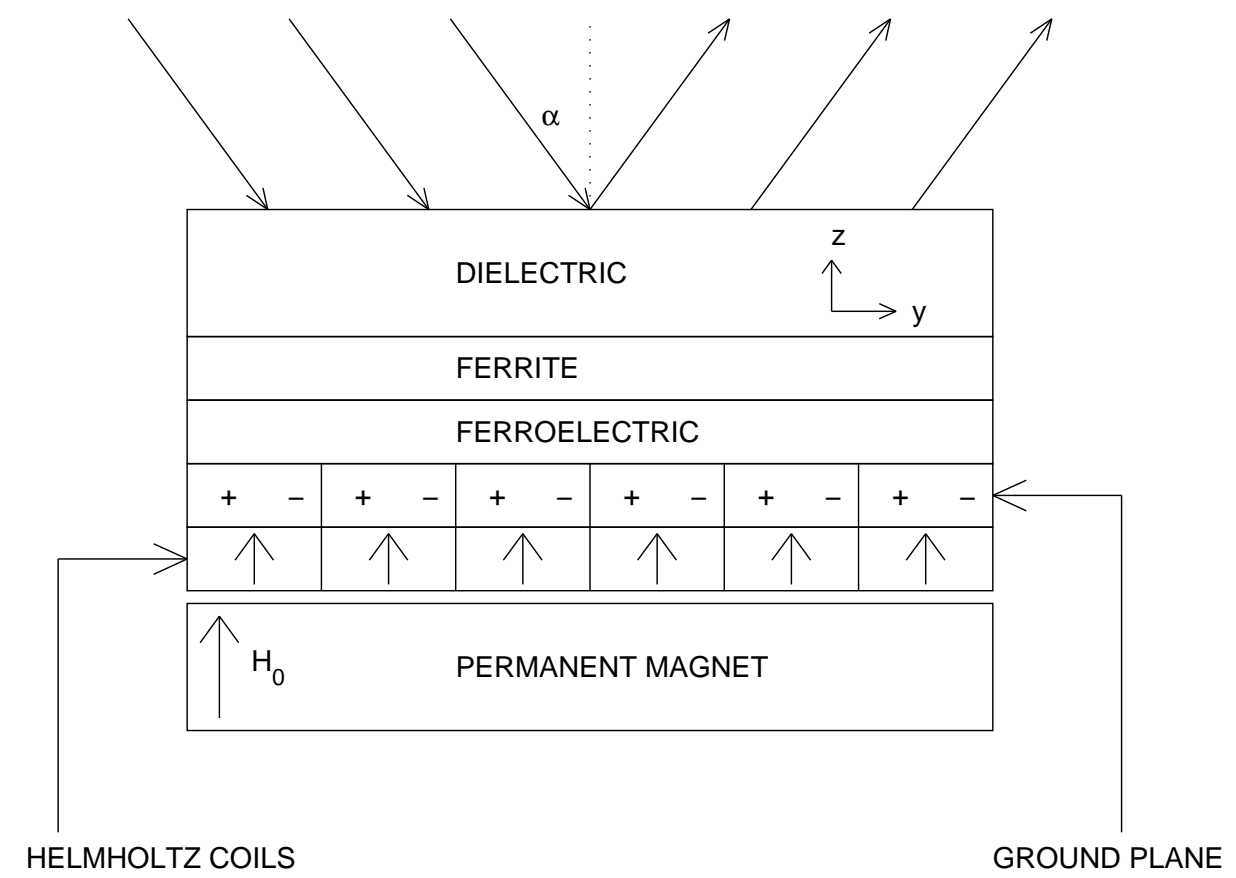

Figure 4: Composite sublayers comprising an active reflector device.

\section{Plane wave solution}

We next discuss a plane wave solution as it interacts with a ferrite layer. Due to the tensor magnetic permeability $\bar{\mu}$, the electric and magnetic modes are coupled in the ferrite layer. In this section we present the detailed calculations for constructing the fundamental solution in the ferrite layer. A similar calculation can be carried out for the ferroelectric layer. 
The time-harmonic Maxwell's equations are written as

$$
\begin{aligned}
\nabla \times H & =i \omega \epsilon E \\
\nabla \times E & =-i \omega \bar{\mu} H \\
\nabla \cdot(\bar{\mu} H) & =0 \\
\nabla \cdot E & =0 .
\end{aligned}
$$

For the ferrite layer the permeability $\bar{\mu}$ is the tensor defined by (3.1), and thus the first three equations in (4.1) can be written as

$$
\begin{gathered}
\left(\begin{array}{c}
\frac{\partial}{\partial y} H_{z}-\frac{\partial}{\partial z} H_{y} \\
\frac{\partial}{\partial z} H_{x}-\frac{\partial}{\partial x} H_{z} \\
\frac{\partial}{\partial x} H_{y}-\frac{\partial}{\partial y} H_{x}
\end{array}\right)=i \omega \epsilon\left(\begin{array}{c}
E_{x} \\
E_{y} \\
E_{z}
\end{array}\right), \\
\left(\begin{array}{c}
\frac{\partial}{\partial y} E_{z}-\frac{\partial}{\partial z} E_{y} \\
\frac{\partial}{\partial z} E_{x}-\frac{\partial}{\partial x} E_{z} \\
\frac{\partial}{\partial x} E_{y}-\frac{\partial}{\partial y} E_{x}
\end{array}\right)=-i \omega\left(\begin{array}{c}
\mu H_{x}-i \kappa H_{z} \\
\mu_{0} H_{y} \\
i \kappa H_{x}+\mu H_{z},
\end{array}\right),
\end{gathered}
$$

and

$$
-\mu\left(\frac{\partial}{\partial x} H_{x}+\frac{\partial}{\partial z} H_{z}\right)=i \kappa\left(\frac{\partial}{\partial z} H_{x}-\frac{\partial}{\partial x} H_{z}\right)+\mu_{0} \frac{\partial}{\partial y} H_{y} .
$$

Taking the cross-product of (4.2) and using (4.3), we obtain (y-component)

$$
i \omega \mu_{0} H_{y}=\frac{1}{i \omega \epsilon}\left(\frac{\partial^{2}}{\partial x^{2}} H_{y}+\frac{\partial^{2}}{\partial z^{2}} H_{y}-\frac{\partial}{\partial y}\left(\frac{\partial}{\partial x} H_{x}+\frac{\partial}{\partial z} H_{z}\right)\right) .
$$

Using the $y$-component of (4.2),

$$
i \omega \epsilon E_{y}=\frac{\partial}{\partial z} H_{x}-\frac{\partial}{\partial x} H_{z}
$$

in (4.4) and substituting into (4.5), we have

$$
\frac{\partial^{2}}{\partial x^{2}} H_{y}+\frac{\partial^{2}}{\partial z^{2}} H_{y}+\frac{\mu_{0}}{\mu} \frac{\partial^{2}}{\partial y^{2}} H_{y}+\omega^{2} \mu_{0} \epsilon H_{y}=\frac{\omega \kappa \epsilon}{\mu} \frac{\partial}{\partial y} E_{y} .
$$


The components of (4.3) can be manipulated to yield the following equations

$$
\begin{aligned}
& \mu\left(\frac{\partial}{\partial y} E_{z}-\frac{\partial}{\partial z} E_{y}\right)+i \kappa\left(\frac{\partial}{\partial x} E_{y}-\frac{\partial}{\partial y} E_{x}\right)=-i \omega\left(\mu^{2}-\kappa^{2}\right) H_{x} \\
& \frac{\partial}{\partial z} E_{x}-\frac{\partial}{\partial x} E_{z}=-i \omega \mu_{0} H_{y} \\
& -i \kappa\left(\frac{\partial}{\partial y} E_{z}-\frac{\partial}{\partial z} E_{y}\right)+\mu\left(\frac{\partial}{\partial x} E_{y}-\frac{\partial}{\partial y} E_{x}\right)=-i \omega\left(\mu^{2}-\kappa^{2}\right) H_{z} .
\end{aligned}
$$

Using the $y$-component of (4.2) and the first and last equations of (4.8), one can obtain

$$
\begin{aligned}
& \frac{\partial}{\partial z}\left(\mu\left(\frac{\partial}{\partial y} E_{z}-\frac{\partial}{\partial z} E_{y}\right)+i \kappa\left(\frac{\partial}{\partial x} E_{y}-\frac{\partial}{\partial y} E_{x}\right)\right) \\
& \quad-\frac{\partial}{\partial x}\left(-i \kappa\left(\frac{\partial}{\partial y} E_{z}-\frac{\partial}{\partial z} E_{y}\right)+\mu\left(\frac{\partial}{\partial x} E_{y}-\frac{\partial}{\partial y} E_{x}\right)\right)=\omega^{2} \epsilon\left(\mu^{2}-\kappa^{2}\right) E_{y} .
\end{aligned}
$$

This can be rearranged to obtain

$$
\begin{aligned}
-\mu & \left(\frac{\partial^{2}}{\partial z^{2}} E_{y}+\frac{\partial^{2}}{\partial x^{2}} E_{y}\right) \\
& +\frac{\partial}{\partial y}\left(\mu\left(\frac{\partial}{\partial z} E_{z}+\frac{\partial}{\partial x} E_{x}\right)-i \kappa\left(\frac{\partial}{\partial z} E_{x}-\frac{\partial}{\partial x} E_{z}\right)\right)=\omega^{2} \epsilon\left(\mu^{2}-\kappa^{2}\right) E_{y} .
\end{aligned}
$$

Using this result with the $y$-component of (4.8) and the fourth equation of (4.1), we have

$$
\frac{\partial^{2}}{\partial x^{2}} E_{y}+\frac{\partial^{2}}{\partial y^{2}} E_{y}+\frac{\partial^{2}}{\partial z^{2}} E_{y}+\frac{\omega^{2} \epsilon\left(\mu^{2}-\kappa^{2}\right)}{\mu} E_{y}=-\frac{\omega \mu_{0} \kappa}{\mu} \frac{\partial}{\partial y} H_{y} .
$$

From (4.2)

$$
i \omega \epsilon\left(\begin{array}{c}
E_{x} \\
E_{z}
\end{array}\right)=\left(\begin{array}{c}
\frac{\partial}{\partial y} H_{z}-\frac{\partial}{\partial z} H_{y} \\
\frac{\partial}{\partial x} H_{y}-\frac{\partial}{\partial y} H_{x}
\end{array}\right) .
$$

If we define

$$
E^{ \pm}=E_{z} \pm i E_{x}, \quad H^{ \pm}=H_{z} \pm i H_{x}
$$

then it follows that

$$
i \omega \epsilon\left(\begin{array}{c}
E^{+} \\
E^{-}
\end{array}\right)=\left(\begin{array}{c}
-i \nabla^{+} H_{y}+i \frac{\partial}{\partial y} H^{+} \\
i \nabla^{-} H_{y}-i \frac{\partial}{\partial y} H^{-}
\end{array}\right)
$$


where

$$
\nabla^{ \pm} \phi=\frac{\partial}{\partial z} \phi \pm i \frac{\partial}{\partial x} \phi
$$

From (4.3)

$$
-i \omega\left(\begin{array}{c}
\mu H_{x}-i \kappa H_{z} \\
\mu H_{z}+i \kappa H_{x}
\end{array}\right)=\left(\begin{array}{c}
\frac{\partial}{\partial y} E_{z}-\frac{\partial}{\partial z} E_{y} \\
\frac{\partial}{\partial x} E_{y}-\frac{\partial}{\partial y} E_{x}
\end{array}\right),
$$

and thus

$$
-i \omega\left(\begin{array}{c}
(\mu+\kappa) H^{+} \\
(\mu-\kappa) H^{-}
\end{array}\right)=\left(\begin{array}{c}
-i \nabla^{+} E_{y}+i \frac{\partial}{\partial y} E^{+} \\
i \nabla^{-} E_{y}-i \frac{\partial}{\partial y} E^{-}
\end{array}\right) .
$$

From (4.10)-(4.11)

$$
-\omega^{2} \epsilon(\mu \pm \kappa) H^{ \pm}-\frac{\partial^{2}}{\partial y^{2}} H^{ \pm} \pm \omega \epsilon \nabla^{ \pm} E_{y}+\frac{\partial}{\partial y} \nabla^{ \pm} H_{y}=0,
$$

and we have

$$
-\omega^{2} \epsilon(\mu \pm \kappa) E^{ \pm}-\frac{\partial^{2}}{\partial y^{2}} E^{ \pm} \mp \omega(\mu \pm \kappa) \nabla^{ \pm} H_{y}+\frac{\partial}{\partial y} \nabla^{ \pm} E_{y}=0 .
$$

Let $\hat{E}$ and $\hat{H}$ be the (partial) Fourier transform (in $(x, y))$ of $E$ and $H$, i.e.,

$$
\hat{E}\left(k_{x}, k_{y}, z\right)=\frac{1}{2 \pi} \int_{-\infty}^{\infty} \int_{-\infty}^{\infty} E(x, y, z) e^{-i k_{x} x-i k_{y} y} d x d y .
$$

Then (4.9) and (4.7) can be written, respectively as

$$
\begin{aligned}
& \frac{\partial^{2}}{\partial z^{2}} \hat{E}_{y}-\left(k_{x}^{2}+k_{y}^{2}-\omega^{2} \epsilon \frac{\mu^{2}-\kappa^{2}}{\mu}\right) \hat{E}_{y}=-i \omega \mu_{0} k_{y} \frac{\kappa}{\mu} \hat{H}_{y} \\
& \frac{\partial^{2}}{\partial z^{2}} \hat{H}_{y}-\left(k_{x}^{2}+\frac{\mu_{0}}{\mu} k_{y}^{2}-\omega^{2} \mu_{0} \epsilon\right) \hat{H}_{y}=i \omega \epsilon k_{y} \frac{\kappa}{\mu} \hat{E}_{y}
\end{aligned}
$$

and (4.10)-(4.11) as

$$
\begin{aligned}
& \hat{E}^{ \pm}=\frac{-i k_{y} \nabla^{ \pm} \hat{E}_{y} \pm \omega(\mu \pm \kappa) \nabla^{ \pm} \hat{H}_{y}}{k_{y}^{2}-\omega^{2} \epsilon(\mu \pm \kappa)} \\
& \hat{H}^{ \pm}=\frac{-i k_{y} \nabla^{ \pm} \hat{H}_{y} \mp \omega \epsilon \nabla^{ \pm} \hat{E}_{y}}{k_{y}^{2}-\omega^{2} \epsilon(\mu \pm \kappa)}
\end{aligned}
$$


where

$$
\nabla^{ \pm}=\frac{\partial}{\partial z} \mp k_{x}
$$

Thus the electric and magnetic modes are coupled by (4.12). Next we find the fundamental solution within the ferrite layer. We look for a solution satisfying

$$
\hat{E}_{y}=i \eta \hat{H}_{y}
$$

Then $\eta$ must satisfy

$$
k_{y}^{2}-\omega^{2} \epsilon \frac{\mu^{2}-\kappa^{2}}{\mu}-\omega \mu_{0} k_{y} \frac{\kappa}{\mu \eta}=\frac{\mu_{0}}{\mu} k_{y}^{2}-\omega^{2} \mu_{0} \epsilon-\omega \epsilon k_{y} \frac{\kappa}{\mu} \eta .
$$

Equation (4.15) has two roots, which we designate as $\eta_{+}$and $\eta_{-}$. Then $\hat{H}_{y}$ must satisfy

$$
\frac{\partial^{2}}{\partial z^{2}} \hat{H}_{y}-\left(k_{x}^{2}+\frac{\mu_{0}}{\mu} k_{y}^{2}-\omega^{2} \mu_{0} \epsilon-\eta_{ \pm} \omega \epsilon k_{y} \frac{\kappa}{\mu}\right) \hat{H}_{y}=0 .
$$

Thus, $\hat{E}_{y}$ and $\hat{H}_{y}$ can be written in the form

$$
\begin{gathered}
\hat{E}_{y}=i \eta_{+}\left(A_{1} e^{i k_{+} z}+B_{1} e^{-i k_{+} z}\right)+i \eta_{-}\left(A_{2} e^{i k_{-} z}+B_{2} e^{-i k_{-} z}\right) \\
\hat{H}_{y}=A_{1} e^{i k_{+} z}+B_{1} e^{-i k_{+} z}+A_{2} e^{i k_{-} z}+B_{2} e^{-i k_{-} z}, \\
k_{ \pm}=\sqrt{\omega^{2} \mu_{0} \epsilon+\eta_{ \pm} \omega \epsilon k_{y} \frac{\kappa}{\mu}-k_{x}^{2}-\frac{\mu_{0}}{\mu} k_{y}^{2}} .
\end{gathered}
$$

Formulae (4.12)-(4.16) are given in $[11,19]$ without detailed derivations. These results allow us to construct the plane wave solution in a ferrite layer.

Moreover, in a general dielectric (including ambient) medium the fundamental solutions (in the partial Fourier domain formulation) are given by

$$
\left(\bar{E}_{x}, 0,0\right) e^{i k_{z} z}, \quad\left(0,-\frac{k_{z}}{\omega \mu} \bar{E}_{x}, \frac{k_{y}}{\omega \mu} \bar{E}_{x}\right) e^{i k_{z} z}
$$

for a perpendicular polarized $\left(\mathrm{TM}_{x}\right.$ mode) incident wave and

$$
\left(0, \frac{k_{z}}{\omega \epsilon} \bar{H}_{x},-\frac{k_{y}}{\omega \epsilon} \bar{H}_{x}\right) e^{i k_{z} z}, \quad\left(\bar{H}_{x}, 0,0\right) e^{i k_{z} z} .
$$


for a parallel polarized ( $\mathrm{TE}_{x}$ mode) incident wave, where $\bar{E}_{x}$ and $\bar{H}_{x}$ are constants, when $k_{x}=0$ and $k_{y}$ are fixed.

Now we consider the case when a ferrite layer with thickness $d$ on a perfectly conducting is impinged upon by the parallel polarized incident wave $(4.17) \vec{H}^{(i)}(x, y, z)=\left(H_{x}^{(i)}, 0,0\right) e^{i \vec{k} \cdot \vec{x}}$ (its partial Fourier transform is $\left.\left(H_{x}^{(i)}, 0,0\right) e^{i k_{z} z}\right)$ with $k_{x}=0$ and $k_{y}$ fixed. The transmitted wave in the ferrite layer is generally not $\mathrm{TE}_{x}$-mode alone and thus has a nontrivial $\hat{E}_{x}^{(t)}$. Hence, for a given incident wave, the reflected wave $\left(\vec{E}^{(r)}, \vec{H}^{(r)}\right) e^{-i k_{z} z}$ in the ambient layer is a linear combination of the two fundamental solutions of the form (4.17)-(4.18) (with $\left.k_{z}=-k_{z}\right)$ and the constant weights $\left(E_{x}^{(r)}, H_{x}^{(r)}\right)$ for the reflected wave can be determined by the system of equations

$$
\left\{\begin{array}{l}
H_{x}^{(i)}+H_{x}^{(r)}=\hat{H}_{x}^{(t)}(0) \\
\frac{k_{z}}{\omega \mu}\left(0+E_{x}^{(r)}\right)=\hat{H}_{y}^{(t)}(0) \\
0+E_{x}^{(r)}=\hat{E}_{x}^{(t)}(0) \\
\frac{k_{z}}{\omega \epsilon}\left(H_{x}^{(i)}-H_{x}^{(r)}\right)=\hat{E}_{y}^{(t)}(0) \\
\hat{E}_{x}^{(t)}(-d)=\hat{E}_{y}^{(t)}(-d)=0
\end{array}\right.
$$

for $\left(A_{1}, B_{1}, A_{2}, B_{2}, E_{x}^{(r)}, H_{x}^{(r)}\right)$. The first two equations impose the continuity of $H$ components, the next two equations impose the continuity of $E$ components at $z=0$ (the interface between the ambient and the ferrite layer), and the last equation enforces the perfectly conducting boundary conditions at $z=-d$. Moreover, the induced surface current $\vec{J}$ due to the incident wave is given by

$$
\vec{J}=\left(\hat{H}_{y}(-d),-\hat{H}_{x}(-d), 0\right)=\vec{n} \times \overrightarrow{\hat{H}}
$$

This construction procedure can be readily extended to the case of the composite of sublayers. 


\subsection{Dielectric case (Fresnel's Law)}

In this section we consider the dielectric layer $\left(\mu=\mu_{0}, \kappa=0\right.$ and thus $\left.\eta=0\right)$ with thickness $d$ on the perfectly conducting medium and show that equation (4.19) reduces to usual Fresnel's law for the parallel polarized $\left(\mathrm{TE}_{x}\right)$ incident wave. In this case we have $E_{x}^{(r)}=E_{x}^{(t)}=H_{y}^{(r)}=H_{y}^{(t)}=0$ and

$$
\begin{aligned}
& k_{z}^{(1)}=\sqrt{\omega^{2} \mu_{0} \epsilon^{(1)}-k_{y}^{2}} \\
& k_{+}=k_{-}=k_{z}^{(2)}=\sqrt{\omega^{2} \mu_{0} \epsilon^{(2)}-k_{y}^{2}} .
\end{aligned}
$$

In the dielectric layer we have (in the partial Fourier domain)

$$
\begin{aligned}
& \hat{H}_{x}^{(t)}=H_{+}^{(t)} e^{i k_{z}^{(2)} z}+H_{-}^{(t)} e^{-i k_{z}^{(2)} z} \\
& \hat{E}_{y}^{(t)}=\frac{k_{z}^{(2)}}{\omega \epsilon^{(2)}}\left(H_{+}^{(t)} e^{i k_{z}^{(2)} z}-H_{-}^{(t)} e^{-i k_{z}^{(2)} z}\right) .
\end{aligned}
$$

where $H_{ \pm}^{(t)}$ are constants. Thus, (4.19) becomes

$$
\left\{\begin{array}{l}
H_{x}^{(i)}+H_{x}^{(r)}=H_{+}^{(t)}+H_{-}^{(t)} \frac{k_{z}^{(1)}}{\omega \epsilon^{(1)}}\left(H_{x}^{(i)}-H_{x}^{(r)}\right)=\frac{k_{z}^{(2)}}{\omega \epsilon^{(2)}} H_{+}^{(t)}-\frac{k_{z}^{(2)}}{\omega \epsilon^{(2)}} H_{-}^{(t)} \\
e^{-i k_{z}^{(2)} d} H_{+}^{(t)}-e^{i k_{z}^{(2)} d} H_{-}^{(t)}=0
\end{array}\right.
$$

Hence we obtain Fresnel's law

$$
H_{x}^{(r)}=\frac{\frac{\epsilon^{(2)} k_{z}^{(1)}-\epsilon^{(1)} k_{z}^{(2)}}{\epsilon^{(2)} k_{z}^{(1)}+\epsilon^{(1)} k_{z}^{(2)}}+e^{-2 i k_{z}^{(2)} d}}{1+\frac{\epsilon^{(2)} k_{z}^{(1)}-\epsilon^{(1)} k_{z}^{(2)}}{\epsilon^{(2)} k_{z}^{(1)}+\epsilon^{(1)} k_{z}^{(2)}} e^{-2 i k_{z}^{(2)} d}} H_{x}^{(i)} .
$$

\subsection{Numerical tests}

In this section we demonstrate the feasibility of using the controllable property of the ferrite layer to attenuate reflections. We select $\epsilon_{0}=1, \mu_{0}=10$ and $d=.5$ (normalized). The 
magnetic permeability $\bar{\mu}$ of the ferrite layer is parameterized by

$$
\mu=\mu_{0}\left(0.3+\frac{\beta}{100}\right), \quad \kappa=\mu_{0}\left(-0.01+\frac{\beta}{100}\right), \quad \text { for } 1 \leq \beta \leq 100
$$

where a parameter $\beta$ plays a role of tuning the frequency $\omega_{m}$ in (3.1). In Figure 5 we depict $\left|\left(E_{x}^{(r)}, H_{x}^{(r)}\right)\right|$ as a function of $\beta$ for the three different incident angles $\phi_{0}=40^{\circ}, 45^{\circ}, 50^{\circ}$. This figure establishes the attenuation capability of the ferrite layer that can be achieved by tuning the permeability. In Figure 6 we graph the real and imaginary parts of $H_{x}^{(r)}$ as a function of the frequency for the incident angle $\phi=45^{\circ}$. This reveals the phase shift capability of the controlled ferrite layer.

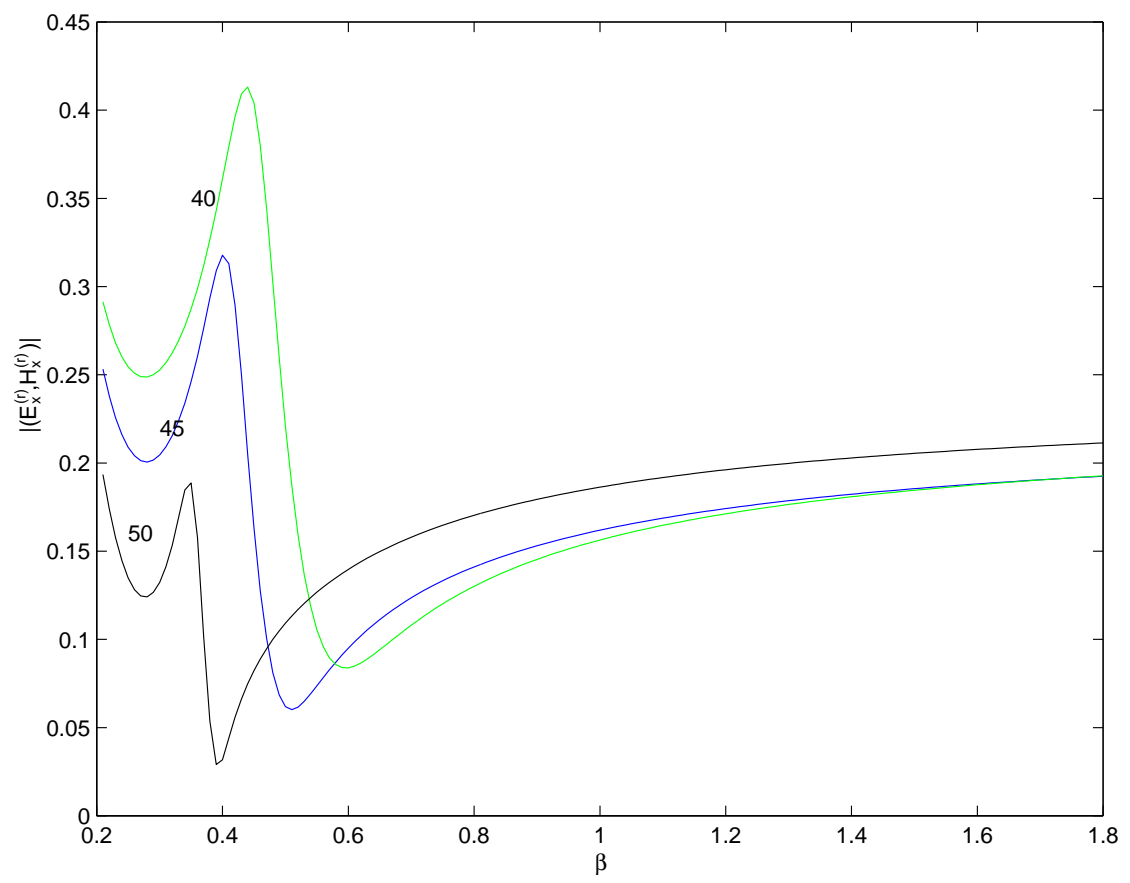

Figure 5: Attenuation Capability

\section{Optimization of material parameters of a coated air- foil}

Having discussed the feasibility of tuning dielectric and magnetic properties of a coating on a perfect conductor in the previous sections, we turn in the next several sections to field 


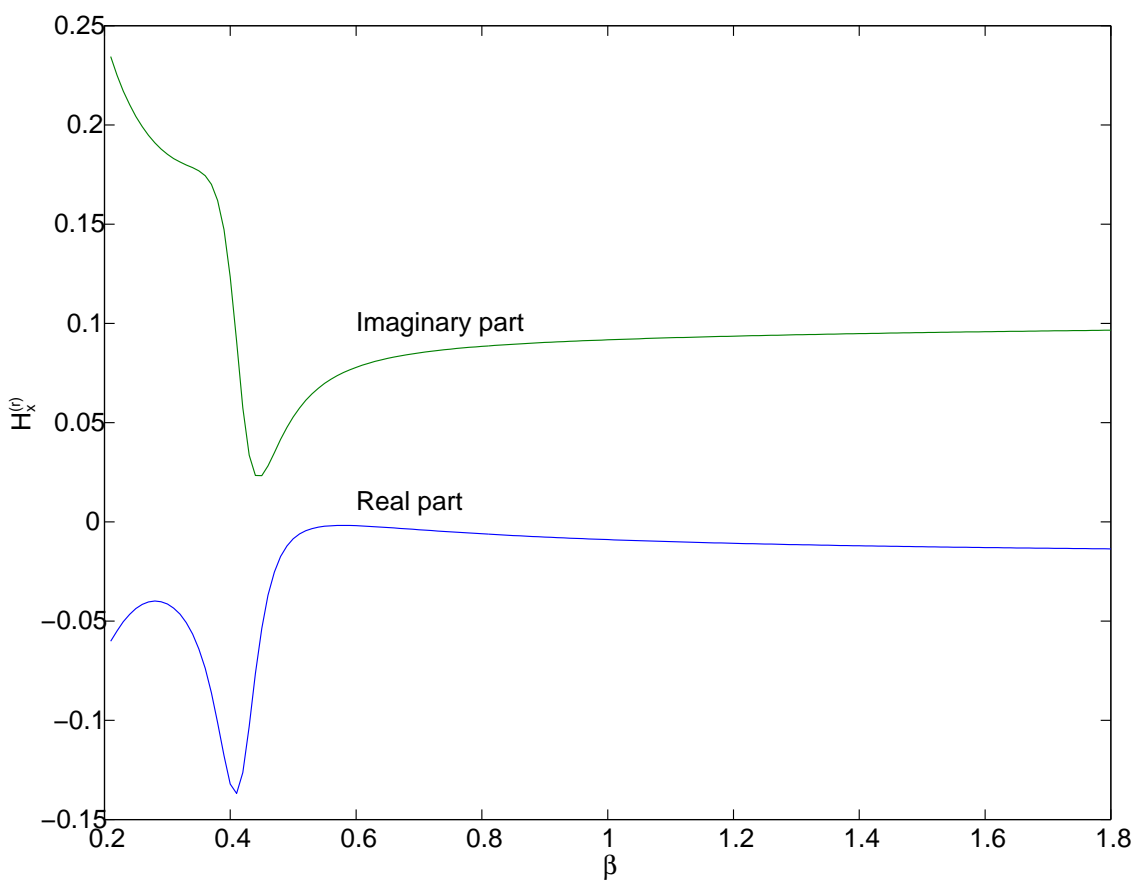

Figure 6: Phase Shift Property

calculations for a coated airfoil and demonstrate our ability to significantly affect the RCS by manipulation of the parameters $\epsilon$ and $\mu$ in the coating.

\subsection{Time-harmonic Maxwell's equation for the transverse mag- netic mode}

We consider the scattering of a perfectly conducting airfoil coated by a layer of constant thickness. The interrogating electromagnetic incident wave is assumed to be a time-harmonic and transverse magnetic, more precisely $\mathrm{TM}_{x}$ mode. Thus, the time-harmonic electric and magnetic fields have the form

$$
E=\left(\begin{array}{c}
E_{x} \\
0 \\
0
\end{array}\right) \quad \text { and } \quad H=-\frac{i}{\omega \mu}\left(\begin{array}{c}
0 \\
\frac{\partial}{\partial z} E_{x} \\
-\frac{\partial}{\partial y} E_{x}
\end{array}\right),
$$

where $E_{x}$ is a function of $y$ and $z$. We denote the airfoil by $\Omega$ and the coating layer by $\Omega_{1}$; see Figure 7. 


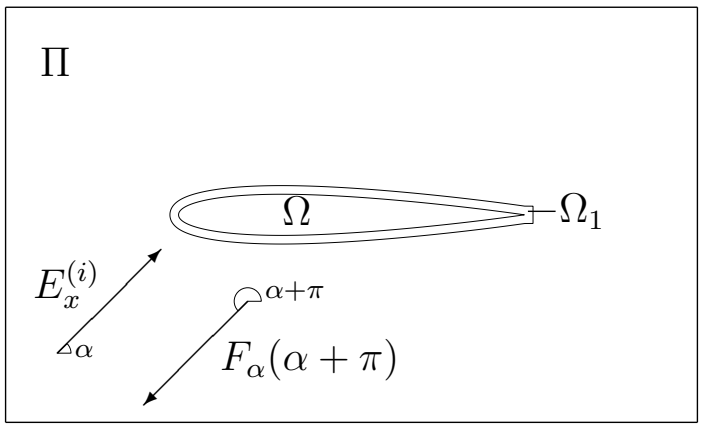

Figure 7: The computational domain, an interrogating wave $E_{x}^{(i)}$ with an interrogation angle $\alpha$, and an far field pattern $F_{\alpha}(\alpha+\pi)$ of a scattered field $E_{x}^{(r)}$ to the direction $\alpha+\pi$.

We decompose the total electric field $E_{x}$ to the incident field $E_{x}^{(i)}$ and the scattered field $E_{x}^{(r)}$, that is, $E_{x}=E_{x}^{(r)}+E_{x}^{(i)}$. Outside the coating layer the interrogating plane wave with an interrogation angle $\alpha=\left(\pi / 2-\phi_{0}\right)$ is given by $E_{x}^{(i)}(y, z)=e^{i\left(k_{y} y+k_{z} z\right)}$, where $k_{y}=k \cos \alpha$, $k_{z}=k \sin \alpha, k=2 \pi / \lambda=\omega / c_{0}$ is the wave number, and $\lambda$ is the wavelength. We do not have an explicit formula for the incident field $E_{x}^{(i)}$ inside the coating layer $\Omega_{1}$. However our following formulation is constructed in such a way that it gives the same total field $E_{x}$ in $\mathbb{R}^{2} \backslash \bar{\Omega}$ and the same scattered field $E_{x}^{(r)}$ in $\mathbb{R}^{2} \backslash\left(\bar{\Omega}_{1} \cup \bar{\Omega}\right)$ regardless how the incident field $E_{x}^{(i)}$ is chosen in the coating layer $\Omega_{1}$. Thus, we can use the same expression $e^{i\left(k_{y} y+k_{z} z\right)}$ for the incident field also in the coating layer.

By eliminating the magnetic field from Maxwell's equation and substituting the time-harmonic electric field $E$ of the form given in (5.1) to the resulting equation, we obtain the following Helmholtz equation

$$
\begin{aligned}
\nabla \cdot\left(\frac{1}{\mu} \nabla E_{x}^{(r)}\right)+\epsilon \omega^{2} E_{x}^{(r)} & =-\nabla \cdot\left(\frac{1}{\mu} \nabla E_{x}^{(i)}\right)-\epsilon \omega^{2} E_{x}^{(i)} & & \text { in } \mathbb{R}^{2} \backslash \bar{\Omega} \\
E_{x}=E_{x}^{(r)}+E_{x}^{(i)} & =0 & & \text { on } \partial \Omega \\
{\left[\frac{1}{\mu} \frac{\partial E_{x}}{\partial n}\right]=\left[E_{x}\right] } & =0 & & \text { on } \partial \Omega_{1} \backslash \partial \Omega \\
\lim _{r \rightarrow \infty} \sqrt{r}\left(\frac{\partial E_{x}^{(r)}}{\partial r}-i k E_{x}^{(r)}\right) & =0, & &
\end{aligned}
$$

where [ • ] denotes the jump and $n$ is a normal direction of the surface $\partial \Omega_{1} \backslash \partial \Omega$. The far field 
behavior of the scattered field described by Maxwell's equation satisfies the Silver-Müller radiation condition [6]. For the time-harmonic $\mathrm{TM}_{x}$ mode this condition reduces to be the Sommerfeld radiation condition given by the limit in (5.2). The material permittivity $\epsilon$ and permeability $\mu$ are piecewise constant functions defined by

$$
\epsilon(y, z)=\left\{\begin{array}{ll}
\epsilon_{r} \epsilon_{0}, & (y, z) \in \bar{\Omega}_{1} \\
\epsilon_{0}, & \text { otherwise }
\end{array} \quad \text { and } \quad \mu(y, z)= \begin{cases}\mu_{r} \mu_{0}, & (y, z) \in \bar{\Omega}_{1} \\
\mu_{0}, & \text { otherwise }\end{cases}\right.
$$

\subsection{Far field pattern}

The far field pattern $F:[0,2 \pi] \rightarrow \mathbb{C}$ describes the intensity and phase of the scattered field $E_{x}^{(r)}$ far away from the scatterer ([5], p.340). It can be defined as

$$
F_{\alpha}(\theta)=\lim _{r \rightarrow \infty}\left(\sqrt{8 \pi k r} e^{-i(k r+\pi / 4)} E_{x}^{(r)}(r \cos \theta, r \sin \theta)\right)
$$

where we have added the subscript $\alpha$ to denote the interrogation angle.

\subsection{Backscatter reduction}

In this optimization problem, we want to find constant material parameters $\epsilon_{r}$ and $\mu_{r}$ so

that the intensity of the backscattered wave is minimized over a given sector $\left[\alpha_{0}, \alpha_{1}\right]$. The objective function is the integral

$$
J\left(\epsilon_{r}, \mu_{r}\right)=\int_{\alpha_{0}}^{\alpha_{1}}\left|F_{\alpha}(\alpha+\pi)\right|^{2} d \alpha
$$

The minimization problem is given by

$$
\min _{\left(\epsilon_{r}, \mu_{r}\right) \in Q} J\left(\epsilon_{r}, \mu_{r}\right)
$$

where $Q$ is the set of admissible material parameters.

This objective function corresponds to a situation where the same radar is illuminating and detecting the scattered wave. The interrogation angle of the interrogating wave varies within 
the interval $\left[\alpha_{0}, \alpha_{1}\right]$ and the formulation (5.4) corresponds to an assumption of a uniform distribution on possible angles of interrogation.

We use the NAG Fortran library's [17] E04UCF implementation of a sequential quadratic programming (SQP) method. This is a gradient based optimization method which approximates the gradient using finite differences.

\section{Approximation}

\subsection{Truncation of domain and variational formulation}

For the discretization of (5.2), we restrict the problem to a rectangular domain $\Pi$ and impose a second-order absorbing boundary condition [1] on the artificial boundary $\partial \Pi$ to

approximate the the Sommerfeld radiation condition. Now the scattered field $E_{x}^{(r)}$ satisfies the following equations

$$
\begin{aligned}
\nabla \cdot\left(\frac{1}{\mu} \nabla E_{x}^{(r)}\right)+\epsilon \omega^{2} E_{x}^{(r)} & =-\nabla \cdot\left(\frac{1}{\mu} \nabla E_{x}^{(i)}\right)-\epsilon \omega^{2} E_{x}^{(i)} & & \text { in } \Pi \backslash \bar{\Omega} \\
E_{x}^{(r)} & =-E_{x}^{(i)} & & \text { on } \partial \Omega \\
{\left[\frac{1}{\mu} \frac{\partial E_{x}}{\partial n}\right]=\left[E_{x}\right] } & =0 & & \text { on } \partial \Omega_{1} \backslash \partial \Omega \\
\frac{\partial E_{x}^{(r)}}{\partial n}-i k E_{x}^{(r)}-\frac{i}{2 k} \frac{\partial^{2} E_{x}^{(r)}}{\partial s^{2}} & =0 & & \text { on } \partial \Pi \\
\frac{\partial E_{x}^{(r)}}{\partial s}-i k \frac{3}{2} E_{x}^{(r)} & =0 & & \text { at } C,
\end{aligned}
$$

where $n$ and $s$ denote the normal and tangential directions of the boundary $\partial \Pi$, respectively, and $C$ is the set of the corner points of $\Pi$.

The variational formulation of $(6.1)$ is : 
Find $E \in\left\{v \in H^{1}(\Pi \backslash \bar{\Omega})|v|_{\partial \Pi} \in H^{1}(\partial \Pi), v=-E_{x}^{(i)}\right.$ on $\left.\partial \Omega\right\}$ such that

$$
\begin{aligned}
& \int_{\Pi \backslash \bar{\Omega}}\left(\frac{1}{\mu} \nabla E_{x}^{(r)} \cdot \nabla v-\epsilon \omega^{2} E_{x}^{(r)} v\right) d \xi+\frac{i}{k \mu_{0}} \int_{\partial \Pi}\left(\frac{1}{2} \frac{\partial E_{x}^{(r)}}{\partial s} \frac{\partial v}{\partial s}-k^{2} E_{x}^{(r)} v\right) d \sigma \\
& +\frac{3}{4 \mu_{0}} \sum_{(y, z) \in C} E_{x}^{(r)}(y, z) v(y, z)=-\int_{\Pi \backslash \bar{\Omega}}\left(\frac{1}{\mu} \nabla E_{x}^{(i)} \cdot \nabla v-\epsilon \omega^{2} E_{x}^{(i)} v\right) d \xi
\end{aligned}
$$

for all $v \in\left\{v \in H^{1}(\Pi \backslash \bar{\Omega})|v|_{\partial \Pi} \in H^{1}(\partial \Pi), v=0\right.$ on $\left.\partial \Omega\right\}$.

\subsection{Finite element approximation}

The finite element approximation is implemented using linear elements. The mesh is constructed from two uniform triangular meshes. The finer mesh is for the coating layer and, the coarser is for the exterior domain outside the coating. The mesh step sizes are chosen in such a way that the number of nodes per wavelength is approximately the same in the air and coating. The finer mesh is locally fitted to the surfaces of the obstacle and the coating layer. The local fitting is done using the algorithm [4] with slight modifications. Between the meshes, there is a layer, which fits the meshes together in a conforming way. An example of a part of mesh is shown in Figure 8.

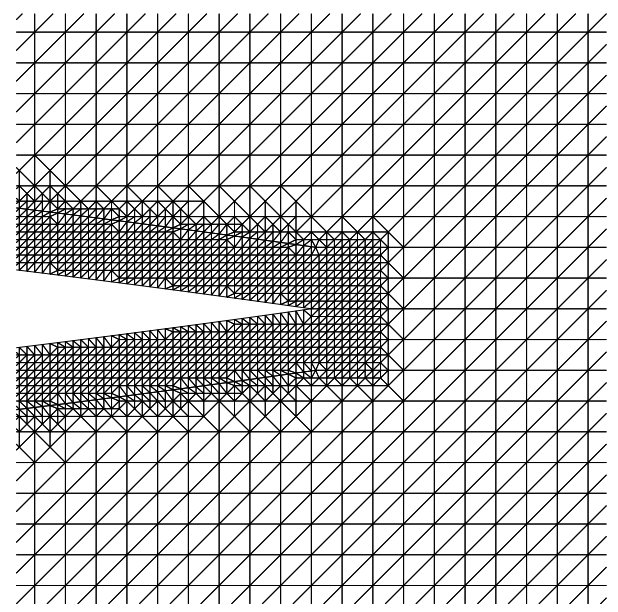

Figure 8: A magnified view of the mesh for a coated NACA0012 airfoil.

After the discretization of the variational formulation (6.2), we obtain a system of linear 
equations

$$
A x=b,
$$

where the matrix $A$ is a symmetric non-Hermitian complex matrix. The vector $x$ contains the nodal values of the scattered field $E_{x}^{(r)}$ and the vector $b$ corresponds the right-hand terms in (6.2). The resulting systems of linear equations are solved using an iterative method which combines fictitious domain and domain decomposition methods [8].

The far field pattern $F_{\alpha}(\alpha+\pi)$ in (5.3) is computed as a surface integral [6] of the computed near field $E_{x}^{(r)}$ and its flux. Our particular implementation of the computations is described in [7]. We need to evaluate the far field pattern $F_{\alpha}(\alpha+\pi)$ in (5.3) using the computed near field $E_{x}^{(r)}$. Our particular implementation of the computations is described in [7]. The basic idea of this procedure is the following. Let $\tilde{E}_{x}^{(r)}$ be the harmonic extension of $E_{x}^{(r)}$ from $\Pi \backslash\left(\bar{\Omega} \cup \Omega_{1}\right)$ to $\mathbb{R}^{2}$. We obtain this extension as a byproduct of our solution procedure. Then at the point $\eta$ outside $\bar{\Omega} \cup \Omega_{1}$ the scattered field $\tilde{E}_{x}^{(r)}$ is given by

$$
\tilde{E}_{x}^{(r)}(\eta)=\int_{\Pi}\left(\Delta \tilde{E}_{x}^{(r)}(\xi)+k^{2} \tilde{E}_{x}^{(r)}(\xi)\right) \Phi(\eta, \xi) d \xi
$$

where $\Phi(\eta, \xi)$ is the fundamental solution of the homogeneous Helmholtz equation in $\mathbb{R}^{2}$ given by the Hankel function $\Phi(\eta, \xi)=\frac{i}{4} H_{0}^{(1)}(k|\eta-\xi|)$. By using Green's formula one can show that (6.4) is equivalent to

$$
\tilde{E}_{x}^{(r)}(\eta)=\int_{\partial \Pi}\left(E_{x}^{(r)}(\sigma) \frac{\partial \Phi(\eta, \sigma)}{\partial n}-\frac{\partial E_{x}^{(r)}(\sigma)}{\partial n} \Phi(\eta, \sigma)\right) d \sigma
$$

which is a more traditional expression for $\tilde{E}_{x}^{(r)}(\eta)[6]$. The far field pattern is obtained by first discretizing the Helmholtz operator and integral in (6.4) and then taking the limit

$$
F_{\alpha}(\alpha+\pi)=\lim _{r \rightarrow \infty}\left(\sqrt{8 \pi k r} e^{-i(k r+\pi / 4)} \tilde{E}_{x}^{(r)}(r \cos (\alpha+\pi), r \sin (\alpha+\pi))\right) .
$$

At the discrete level the previous procedure reduces to the evaluation of a sum of exponential functions. This can be performed easily and fast. 
We have compared numerical results computed using the proposed method and the same implementation with test cases presented on a workshop in Oxford [18]. The two-dimensional test cases did not include a coated NACA airfoil, but they did include a similar coated ogive (the intersection of two non-concentric disks). We computed numerical results for 10 test cases. All our numerical results and especially the radar cross sections were in very good agreement with the majority of the results presented in the workshop for each test case. Because of this, we expect our results to be accurate also for the coated NACA airfoils.

\section{Numerical experiments}

In our experiments, we minimize the backscatter by a coated NACA0012 airfoil. The length of the airfoil is one unit without coating and the trailing edge of the perfectly conducting material is at the origin. We minimize the backscatter for the interrogation angles in the sector $\left[\alpha_{0}, \alpha_{1}\right]=[0, \pi]$. We considered two wavelengths, $\lambda=1 / 4$ and $\lambda=1 / 10$. The thickness of the coating is $\lambda / 10$.

We consider first the lower frequency experiments with a four wavelength long airfoil. The computational domain is $[-1.5,0.5] \times[-0.6,0.6]$. Our discretization has 20 nodes per wavelength in the ambient medium leading to a triangulation with 22157 nodes and 43158 elements. A magnified view of the mesh at the trailing edge is shown in Figure 8. We perform several optimizations with different box constraints for the real and imaginary parts of $\epsilon_{r}$ and $\mu_{r}$. The results of these optimizations are given in Table 1. A surface plot of the objective function is shown in Figure 9. The radar cross sections defined by

$$
\operatorname{RCS}(\alpha)=10 \log _{10}\left(\frac{1}{8 \pi}\left|F_{\alpha}(\alpha+\pi)\right|^{2}\right)
$$

are shown for two optimized materials in Figure 10. Corresponding reflected field intensities for comparison between the no coating layer case and the optimized complex-valued parameters case of Figure 10 are depicted in Figures 11 and 12 for an angle of interrogation 


\begin{tabular}{c|ccc|ccc|c} 
& \multicolumn{3}{|c|}{ Real part } & \multicolumn{3}{|c|}{ Imaginary part } & \multirow{2}{*}{ ( $\left.\min , \mu_{r}\right)$} \\
\hline$\epsilon_{r}$ & 1 & 1 & 1 & 0 & 0 & 0 & \\
$\mu_{r}$ & 1 & 1 & 1 & 0 & 0 & 0 & 324.340 \\
\hline$\epsilon_{r}$ & 1 & 3.92 & 10 & 0 & 0 & 0 & \\
$\mu_{r}$ & 1 & 1 & 10 & 0 & 0 & 0 & 318.738 \\
\hline$\epsilon_{r}$ & 1 & 6.52 & 10 & 0 & 3.14 & 10 & \\
$\mu_{r}$ & 1 & 1 & 1 & 0 & 0 & 0 & 2.918 \\
\hline$\epsilon_{r}$ & 1 & 7.75 & 10 & 0 & 0.80 & 10 & \\
$\mu_{r}$ & 1 & 5.41 & 10 & 0 & 2.51 & 10 & 0.083
\end{tabular}

Table 1: The results of material parameter optimizations for the case $\lambda=1 / 4$.

\begin{tabular}{c|ccc|ccc|c} 
& \multicolumn{3}{|c|}{ Real part } & \multicolumn{3}{|c|}{ Imaginary part } & \multirow{2}{*}{ ( $\left.\min , \mu_{r}\right)$} \\
\hline$\epsilon_{r}$ & 1 & 1 & 1 & 0 & 0 & 0 & \\
$\mu_{r}$ & 1 & 1 & 1 & 0 & 0 & 0 & 806.036 \\
\hline$\epsilon_{r}$ & 1 & 3.84 & 10 & 0 & 0 & 0 & \\
$\mu_{r}$ & 1 & 1 & 10 & 0 & 0 & 0 & 802.695 \\
\hline$\epsilon_{r}$ & 1 & 6.58 & 10 & 0 & 3.14 & 10 & \\
$\mu_{r}$ & 1 & 1 & 1 & 0 & 0 & 0 & 2.901 \\
\hline$\epsilon_{r}$ & 1 & 4.54 & 10 & 0 & 2.99 & 10 & \\
$\mu_{r}$ & 1 & 4.99 & 10 & 0 & 2.92 & 10 & 0.234
\end{tabular}

Table 2: The results of material parameter optimizations for the case $\lambda=1 / 10$.

$\alpha=\pi / 4$.

The computational domain for the higher frequency experiments with a ten wavelengths long airfoil is $[-1.3,0.3] \times[-0.4,0.4]$. Again our discretization has 20 nodes per wavelength in the ambient medium leading to a triangulation with 65551 nodes and 128530 elements. The results of optimizations with different box constraints are given in Table 2. Radar cross sections for two optimized materials are shown in Figure 13. Corresponding reflected field intensities for comparison between the no coating layer case and the optimized complexvalued parameters case of Figure 13 are depicted in Figures 14 and 15 for an angle of interrogation $\alpha=\pi / 4$. 


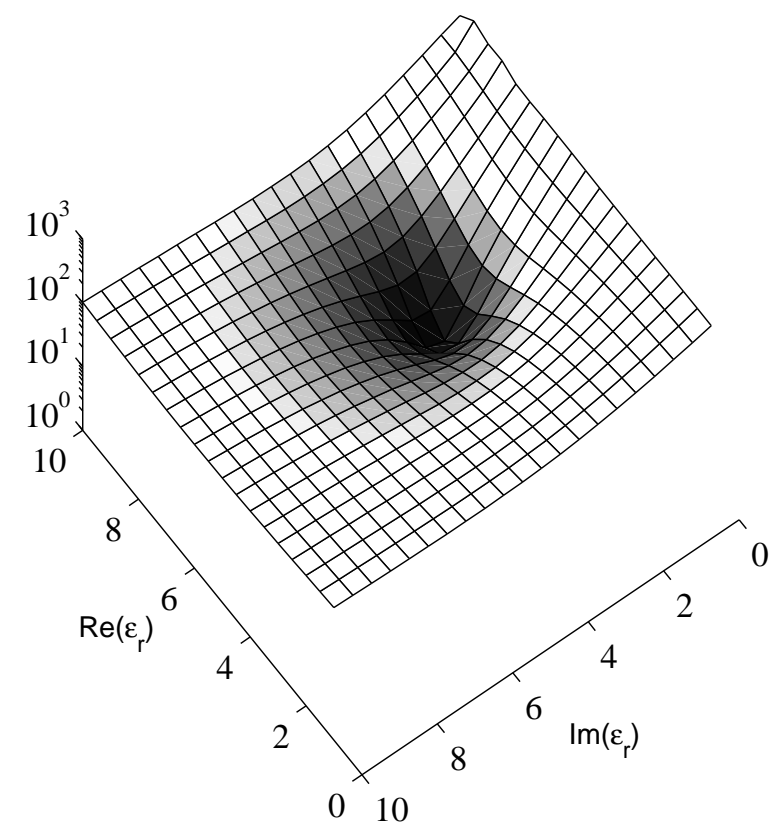

Figure 9: The objective function $J\left(\epsilon_{r}, \mu_{r}\right)$ for complex-valued $\epsilon_{r}$ and $\mu_{r}=1$ for the case $\lambda=1 / 4$.

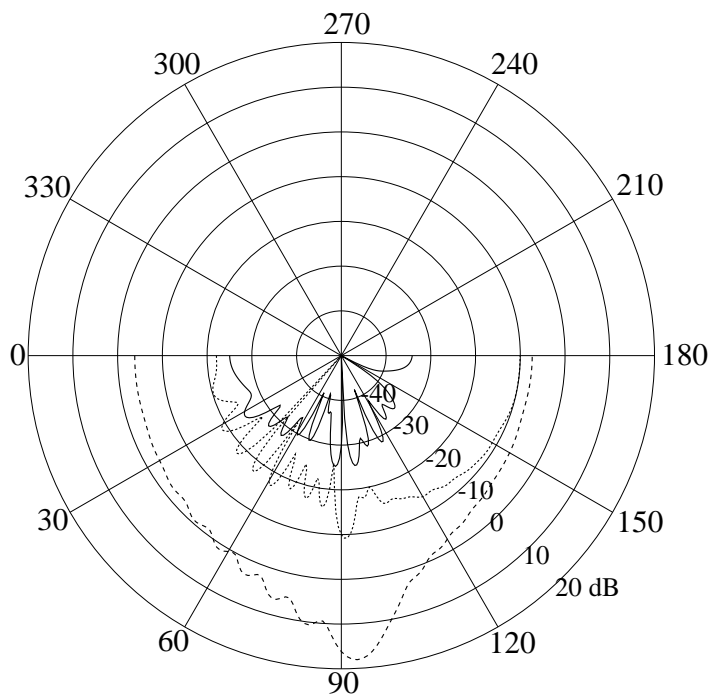

Figure 10: The RCS for optimized complex-valued material parameters $\left(\epsilon_{r}=7.75+0.80 i\right.$; $\mu_{r}=5.41+2.51 i$; solid line $)$, optimized complex-valued permittivity $\left(\epsilon_{r}=6.52+3.14 i\right.$; $\mu_{r}=1$; dotted line), and for no coating $\left(\epsilon_{r}=1 ; \mu_{r}=1\right.$; dashed line) for the case $\lambda=1 / 4$. 


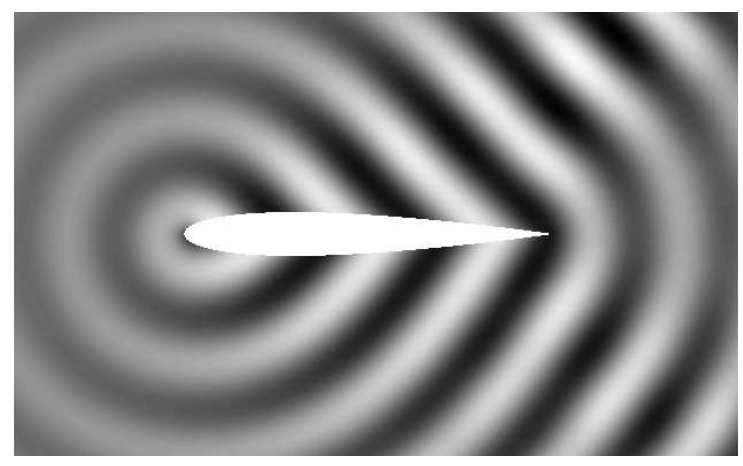

Figure 11: The reflected field $E_{x}^{(r)}$ for no coating $\left(\epsilon_{r}=1 ; \mu_{r}=1\right)$ for the case $\lambda=1 / 4$ and angle of interrogation $\alpha=\pi / 4$.

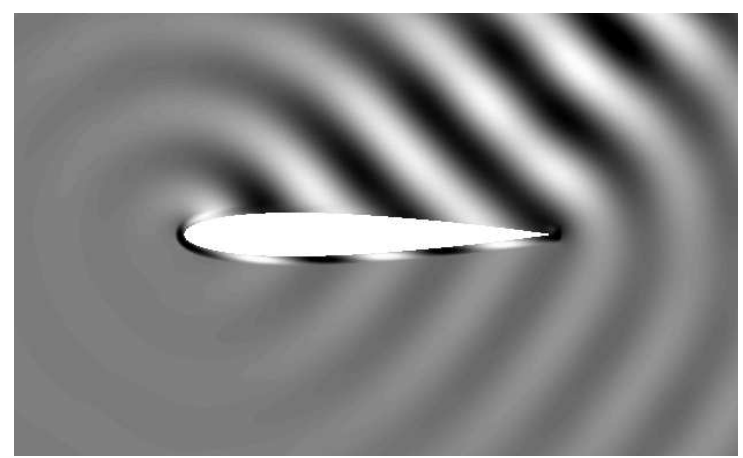

Figure 12: The reflected field $E_{x}^{(r)}$ for optimized complex-valued material parameters $\left(\epsilon_{r}=\right.$ $\left.7.75+0.80 i ; \mu_{r}=5.41+2.51 i\right)$ for the case $\lambda=1 / 4$ and angle of interrogation $\alpha=\pi / 4$.

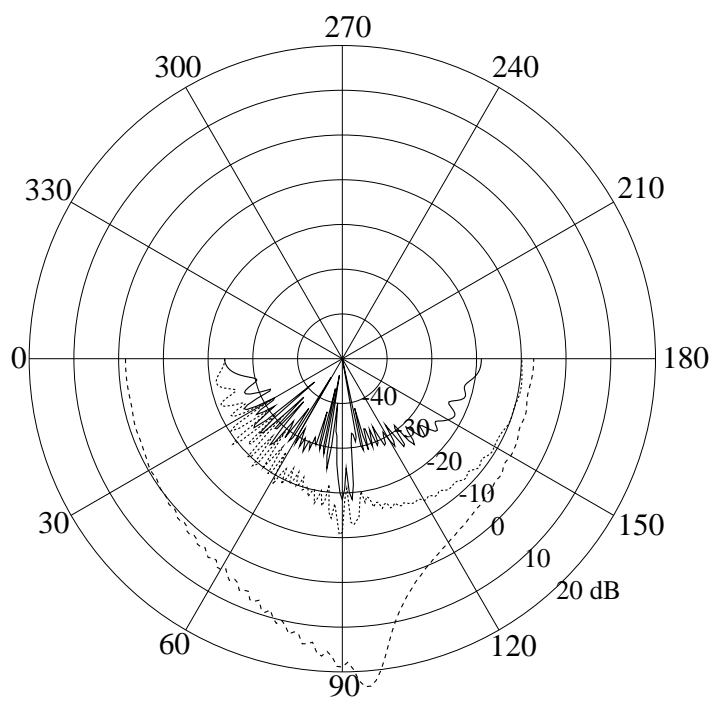

Figure 13: The RCS for optimized complex-valued material parameters $\left(\epsilon_{r}=4.54+2.99 i\right.$; $\mu_{r}=4.99+2.92 ;$ solid line $)$, optimized complex-valued permittivity $\left(\epsilon_{r}=6.58+3.14 i\right.$; $\mu_{r}=1$; dotted line), and for no coating $\left(\epsilon_{r}=1 ; \mu_{r}=1\right.$; dashed line) for the case $\lambda=1 / 10$. 


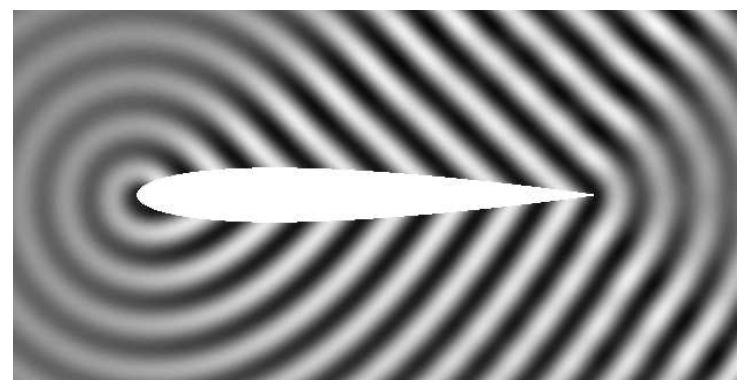

Figure 14: The reflected field $E_{x}^{(r)}$ for no coating $\left(\epsilon_{r}=1 ; \mu_{r}=1\right)$ for the case $\lambda=1 / 10$ and angle of interrogation $\alpha=\pi / 4$.

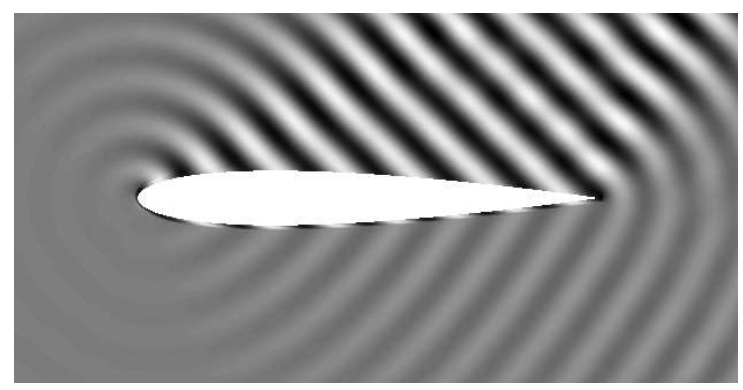

Figure 15: The reflected field $E_{x}^{(r)}$ for optimized complex-valued material parameters $\left(\epsilon_{r}=\right.$ $\left.4.54+2.99 i ; \mu_{r}=4.99+2.92 i\right)$ for the case $\lambda=1 / 10$ and angle of interrogation $\alpha=\pi / 4$. 


\section{Summary and conclusions}

In summary, the efforts reported on in this paper are an important first step in developing an attenuation or anti-interrogation technology. We considered first the question of the feasibility of reduction in reflected electromagnetic waves from a planar layered coating on a perfect conductor. We demonstrated that even under uncertainty of the interrogating wavelengths (frequencies), one can achieve reduction of the reflection coefficient through optimizing the dielectric permittivity in a coating layer. We then considered a Maxwell equation based formulation for a composite ferromagnetic-ferroelectric device built and experimentally tested by How and Vittoria. We derived the pertinent reflection field equations for time harmonic interrogating $\mathrm{TM}_{x}$ mode plane waves and showed that substantial control of reflected waves (both magnitude and phase) can be obtained by tuning the magnetic permeability of a ferrite layer. We next turned to a non-planar geometry, in this case a 2-D airfoil, with a coating layer wherein both the dielectric permittivity $\epsilon$ and the magnetic permeability $\mu$ can be optimized. Allowing a uniform uncertainty on the interrogating signal angles, we use computational methods from 2-D scattering theory (the Helmholtz equation with Sommerfeld far field radiation conditions) to verify that significant reduction in the far field reflection can be obtained by optimal choice of $\epsilon$ and $\mu$.

All of the investigations discussed in this paper were pursued under an active design scenario and did not allow for online adaptivity of the coating layers. Future investigations of great interest include the feasibility of combining the formulations in this paper with real time sensing and adaptive (feedback) control of coatings such as those described above to develop an active control attenuation capability. 


\section{Acknowledgements}

This research was supported in part by the U.S. Air Force Offices of Scientific Research under grant AFOSR FA9550-04-1-0220. The authors would like to thank Dr. Richard Albanese of the AFRL, Brooks AFB, San Antonio TX, for numerous stimulating and valuable comments and suggestions on this work. They are also grateful to Dr. Hoton How for several helpful conversations.

\section{References}

[1] A. Bamberger, P. Joly, and J. E. Roberts, Second-order absorbing boundary conditions for the wave equation: a solution for the corner problem, SIAM J. Numer. Anal. 27, (1990), 323-352.

[2] H. T. Banks, M. W. Buksas, and T. Lin, Electromagentic Material Interrogation Using Conductive Interfaces and Acoustic Wavefronts, SIAM, Philadelphia, 2000.

[3] S. Beeler, H. T. Tran, and N. Dietz, Representation of GaP formation by a reduced order surface kinetics model using p-polarized reflectance measurements, J. Appl. Phys 86, (1999), 674-682.

[4] C. Börgers, A triangulation algorithm for fast elliptic solvers based on domain imbedding, SIAM J. Numer. Anal. 27, (1990), 1187-1196.

[5] D. Colton, The inverse scattering problem for time-harmonic acoustic waves, SIAM Review 26, (1984), 323-350.

[6] D. Colton and R. Kress, Inverse Acoustic and Electromagnetic Scattering Theory, 2nd ed., Springer-Verlag, New York, 1998. 
[7] E. Heikkola, Yu. A. Kuznetsov, P. Neittaanmäki, and J. Toivanen, Fictitious domain methods for the numerical solution of two-dimensional scattering problems, J. Comp. Phys. 145, (1998), 89-109.

[8] E. Heikkola, T. Rossi, and J. Toivanen, A domain decomposition technique for twodimensional scattering problems with coated obstacles, in Innovative Tools for Scientific Computation in Aeronautical Engineering, J. Periaux, P. Joly, O. Pironneau, E. Onate (eds.), CIMNE, 2001, 183-194.

[9] H. How and C. Vittoria, Implementation of microwave active nulling, private communication

[10] H. How and C. Vittoria, Microwave impedance control over a ferroelectric boundary layer, IEEE Transactions on Microwave Theory and Techniques 52, (2004) 2177-2182.

[11] H. How, X. Zuo, E. Hokanson, L. Kempel and C. Vittoria, Calculated and measured characteristics of a microstrip fabricated on a Y-type hexaferrite substrate, IEEE Transactions on Microwave Theory and Techniques 50, (2002) 1280-1288.

[12] K. Ito and K. Kunisch, Sensitivity analysis of solution to optimization problems in Hilbert spaces with Applications to optimal control and estimation, J. Differential Equation 99, (1992), 1-40.

[13] K. Ito and F. Reitich, A high-order perturbation approach to profile reconstruction. I: Perfectly conducting gratings, Inverse Problems, 15 (1999), 1067-1085.

[14] J. D. Jackson, Classical Electrodynamics, Wiley \& Sons, New York, 1975.

[15] C.L. Ladson, C.W. Brooks Jr., A.S. Hill and D.W. Sproles, Computer Program To Obtain Ordinates for NACA Airfoils, NASA Technical Memorandum 4741, NASA, 1996.

[16] B. Lax and K.J. Button, Microwave Ferrites and Ferromagnetics, McGraw-Hill, New York, 1962. 
[17] The Numerical Algorithms Group Ltd, The NAG Fortran Library Manual: Mark 20, Oxford, U.K., 2002.

[18] IMA (U.K.), GAMNI/SMAI (France) and Oxford University Computing Laboratory (U.K.), Technical Description of Workshop on Approximations and Numerical Methods for the Solution of the Maxwell Equations, Oxford, U.K., 1995.

[19] E.L.B. El-Sharawy and R.W. Jackson, Coplanar waveguide and slot line on magnetic substrates: Analysis and experiment, IEEE Transactions on Microwave Theory and Techniques 36, (1988) 1071-1079. 